\title{
Presentation
}

\section{Thematic section: Psychology and Phenomenology: A field of interlocution in constant transformation}

The contribution of Phenomenology to research and the practice of Psychology in Brazil stems from the effort of many researchers whose scientific production in the form of articles and books have been enriching Phenomenology over the years. Specifically in the last decade, the phenomenological research field in Brazil developed significantly, based on the higher number of publications relating Psychology and Phenomenology, the set of research groups registered at the Conselho Nacional de Desenvolvimento Científico e Tecnológico (CNPq, National Council for Scientific and Technological Development) (about 50), and the number of researchers who link their studies directly to Phenomenology (about 577 according to a recent survey).

The complexity of viewpoints and authors, far from impoverishing the debate, invites reflections, given the plural epistemological context. Intervention and research fields were constructed based on phenomenological and existential tradition, which in turn was based on the works of Edmund Husserl, Martin Heidegger, Edith Stein, Maurice Merleau-Ponty, Martin Buber, Jean-Paul Sartre, Michel Henry, and Emmanuel Lévinas, among others. Thus, the six articles chosen to compose this Thematic Section are to a certain degree seminal points because they allow one to conjecture possibilities in the understanding of human beings in their intense activity of being and relating, while they imprint meanings and sense to their own existence. Whether based on considering the essences of the human phenomenon, or by emphasizing their dimension of being in the world, or even by carefully scrutinizing the way the living world is structured, that which reveals itself to the researcher's mind has the power of deconstructing his uncertainties, instigating him to question his experiences and redirect the sense of his own existence.

In the context of the Associação Nacional de Pós Graduação e Pesquisa em Psicologia (ANPEPP, National Association of Graduate Studies and Research in Psychology), a research group called Psychology and Phenomenology was created in 2014, composed of researchers from fourteen higher education institutions located in nine states representing all regions of the country. This group intends to develop means of intervention, listening, and considering suffering, taking into account the singularities and otherness that constitute human beings, involving the discussion of many spaces that manifest subjectivity. In this respect it is important to resume the sense attributed to clinical psychology, which transcends the traditional concept and becomes understood as the privileged space of listening and caring, of considering and getting in contact with the diversity of manifestations of human subjects in their different ways of experiencing their existence.

In this landscape, Adriano Holanda presents a historic panorama about the precursors of Phenomenology in Brazil and the influence it had on Psychology from the work of Waclaw Radecki and Nilton Campos, as pioneers, in addition to the first references in the field of Philosophy with Euryalo Cannabrava and Vicente Ferreira da Silva. Elza Dutra proposes an approximation between the construct self, as proposed by the North American psychologist Carl Rogers in his theory of personality, and the notion of being-there, 
an idea present in the analytic of human existence developed by the German philosopher Martin Heidegger. Virgínia Moreira considers that the Philosophy of ambiguity by Merleau Ponty constitutes a methodological possibility to describe the psychopathological experience where the singular and the universal meet, having culture as a constitutive dimension. Miguel Mahfoud presents the notion of experience in his own dynamic based on the thought of the Italian philosopher Luigi Giussani, pointing out its originality when considered under the perspective of intelligence of the sense of things that enables the emergence of reality to the mind and that is examined in its characteristic of incrementing one's ability to understand and love, and to open oneself to totality. Florinda Martins and Andrés Antúnez discuss the possibility of understanding humans based on the phenomenality of their therapeutic needs. In this sense phenomenality of hallucination, examined under the light of Michel Henry, allows one to think that the phenomenality of vision, touch, and anguish is comparable to the phenomenality of hallucination. Finally, the article written by Daniela Benites, Gustavo Gaueri, and William Gomes resorts to Semiotic phenomenology to analyze personal diaries in blogs and define their potentialities as a movement that propitiates personal changes and self-innovation.

May this set of reflections derived from creative dialogues between Phenomenology and Psychology contribute to the emergence of new initiatives in the sense of providing careful and pertinent care to human beings in their process of recreating the world based on living dynamically with themselves and others.

Prof. Dr. Vera Engler Cury Associate Editor and Pro Dean of Extension and Community Topics Pontifical Catholic University of Campinas, Center of Life Sciences, Graduate Program in Psychology as Profession and Science 